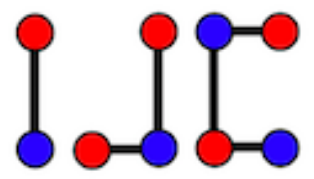

\title{
On the total vertex irregularity strength of comb product of two cycles and two stars
}

\author{
Rismawati Ramdani \\ Department of Mathematics, Faculty of Science and Technology, \\ UIN Sunan Gunung Djati Bandung, Indonesia \\ rismawatiramdani@uinsgd.ac.id
}

\begin{abstract}
Let $G=(V(G), E(G))$ be a graph and $k$ be a positive integer. A total $k$-labeling of $G$ is a map $f: V(G) \cup E(G) \rightarrow\{1,2, \ldots, k\}$. The vertex weight $v$ under the labeling $f$ is denoted by $w_{f}(v)$ and defined by $w_{f}(v)=f(v)+\sum_{u v \in E(G)} f(u v)$. A total $k$-labeling of $G$ is called vertex irregular if there are no two vertices with the same weight. The total vertex irregularity strength of $G$, denoted by $\operatorname{tvs}(G)$, is the minimum $k$ such that $G$ has a vertex irregular total $k$-labeling. This labelings were introduced by Bača, Jendrol̆, Miller, and Ryan in 2007. Let $G$ and $H$ be two connected graphs. Let $o$ be a vertex of $H$. The comb product between $G$ and $H$, denoted by $G \triangleright_{o} H$, is a graph obtained by taking one copy of $\mathrm{G}$ and $|V(G)|$ copies of $H$ and grafting the $i$-th copy of $H$ at the vertex $o$ to the $i$-th vertex of $G$. In this paper, we determine the total vertex irregularity strength of comb product of two cycles and two stars.
\end{abstract}

Keywords: total vertex irregular labeling, total vertex irregularity strength, comb product, cycle, star

Mathematics Subject Classification : 05C78

DOI: $10.19184 /$ ijc.2019.3.2.2

\section{Introduction}

Let $G$ be a graph with vertex set $V(G)$ and edge set $E(G)$. A total labeling $f: V \cup E \rightarrow$ $\{1,2, \ldots, k\}$ is called a vertex irregular total $k$-labeling of $G$ if every two distinct vertices $x$ and $y$ in $V(G)$ satisfy $w t(x) \neq w t(y)$, where $w t(x)=f(x)+\sum_{x z \in E(G)} f(x z)$. The total vertex irregularity strength of $G$, denoted by $\operatorname{tvs}(G)$, is the minimum $k$ for which $G$ has a vertex irregular total $k$ -

Received: 31 May 2019, Revised: 26 Sep 2019, Accepted: 25 Nov 2019. 
labeling. In [1], Bača et al. gave the bounds for a graph $G$ with minimum degree $\delta(G)$ and maximum degree $\Delta(G)$ by the following form:

$$
\lceil(|V(G)|+\delta(G)) /(\Delta(G)+1)\rceil \leq \operatorname{tvs}(G) \leq|V(G)|+\Delta(G)-2 \delta+1 .
$$

In [6], Przybylo proved that $\operatorname{tvs}(G)<32|V(G)| / \delta(G)+8$ in general and $\operatorname{tvs}(G)<8|V(G)| / r+3$ for $r$-regular graphs.

Ramdani et al. in [7], gave an upper bound on the total vertex irregularity strength for $\bigcup_{i=1}^{m} G_{i}$ as follows.

Let $G_{i}$ be an $r$-regular graph, for $i=1,2, \ldots, m$. Then

$$
\operatorname{tvs}\left(\bigcup_{i=1}^{m} G_{i}\right) \leq \sum_{i=1}^{m} \operatorname{tvs}\left(G_{i}\right)-\left\lfloor\frac{m-1}{2}\right\rfloor .
$$

In the same paper, Ramdani et al. obtained the exact value of the total vertex irregularity strength for disjoint union of arbitrary $r$-regular graphs $G_{i}$, for $i=1,2, \ldots, m$, if there is a vertex irregular total $\left(\operatorname{tvs}\left(G_{i}\right)\right)$-labeling of $G_{i}$ such that the vertex-weight function

$$
w_{f_{i}}\left(v_{i a}\right): V\left(G_{i}\right) \rightarrow\left\{r+1, r+2, \cdots,(r+1) \operatorname{tvs}\left(G_{i}\right)-1\right\}
$$

is a bijection for every $i=1,2, \ldots, m$, which is

$$
\operatorname{tvs}\left(\bigcup_{i=1}^{m} G_{i}\right)=\sum_{i=1}^{m} \operatorname{tvs}\left(G_{i}\right)-m+1 \text {. }
$$

In [3], Nurdin proved that

$$
\operatorname{tvs}(G) \geq \max \left\{\left\lceil\frac{\delta+n_{\delta}}{\delta+1}\right\rceil,\left\lceil\frac{\delta+n_{\delta}+n_{\delta+1}}{\delta+2}\right\rceil, \cdots,\left\lceil\frac{\delta+\sum_{i=\delta}^{\Delta} n_{i}}{\Delta+1}\right\rceil\right\}
$$

for connected graph $G$ having $n_{i}$ vertices of degree $i(i=\delta, \delta+1, \delta+2, \cdots, \Delta)$, where $\delta$ and $\Delta$ are the minimum and the maximum degree of $G$, respectively.

In [8], Ramdani and Ramdhani obtained the exact value of the total vertex irregularity strength of comb product between cycles $C_{n}$ and $C_{4}$, as follows.

$$
\operatorname{tvs}\left(C_{n} \triangleright_{o} C_{4}\right)=n+1, \text { for } n \geq 3 \text {. }
$$

Some other results of the total vertex irregularity strength of graphs can be found in [2], [4], [5], [9], and [10].

\section{Main Results}

In this paper we determine the total vertex irregularity strength of some comb product graphs.

Let $o$ be a vertex of $H$. The comb product between $G$ and $H$, denoted by $G \triangleright_{o} H$, is a graph obtained by taking one copy of $\mathrm{G}$ and $|V(G)|$ copies of $H$ and grafting the $i$-th copy of $H$ at the vertex $o$ to the $i$-th vertex of $G$.

An illustration of comb product graph is given in Figure 1. 

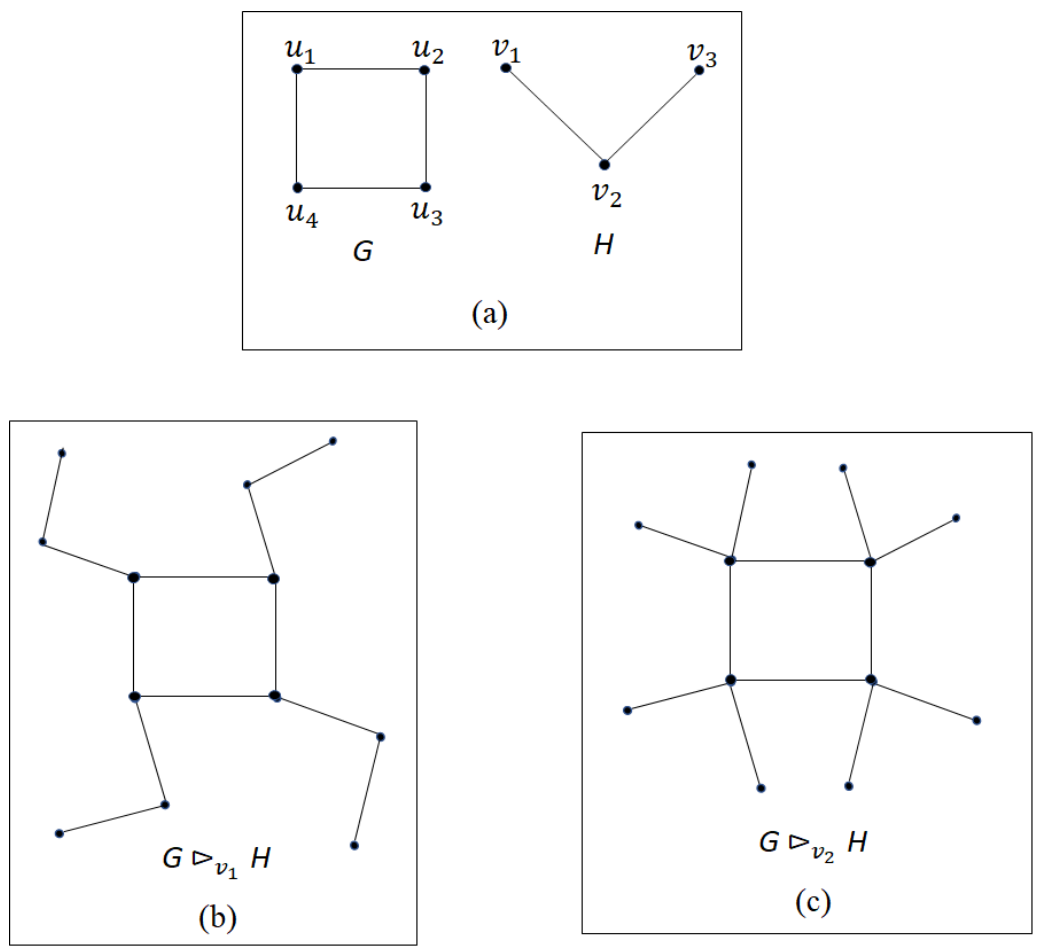

Figure 1. (a) Two graphs $G$ and $H$; (b) The comb product graph $G \triangleright_{v_{1}} H$; (c) The comb product graph $G \triangleright_{v_{2}} H$

The first result gives the total vertex irregularity strength of comb product between two cycles.

Theorem 2.1. Let $C_{m}$ and $C_{n}$ be cycles with order $m$ and $n$ respectively. Then, for $m \geq 3, n \geq 3$, and for every vertex o in $C_{m}$,

$$
\operatorname{tvs}\left(C_{m} \triangleright_{o} C_{n}\right)=\left\lceil\frac{m(n-1)+2}{3}\right\rceil .
$$

Proof. Let $V\left(C_{m} \triangleright_{o} C_{n}\right)=\left\{u_{i, j} \mid 1 \leq i \leq m, 1 \leq j \leq n\right\}$ and

$$
\begin{aligned}
E\left(C_{m} \triangleright_{o} C_{n}\right)= & \left\{u_{i, j} u_{i, j+1} \mid 1 \leq i \leq m, 1 \leq j \leq n-1\right\} \cup\left\{u_{i, n} u_{i, 1} \mid 1 \leq i \leq m\right\} \\
& \cup\left\{u_{i, 1} u_{i+1,1} \mid 1 \leq i \leq m-1\right\} \cup\left\{u_{m, 1} u_{1,1}\right\} .
\end{aligned}
$$

An illustration of $C_{m} \triangleright_{o} C_{n}$ with $o$ be a vertex in $C_{n}$ can be seen in Figure 2.

The $C_{m} \triangleright_{o} C_{n}$ graphs have $m(n-1)$ vertices with degree $\delta=2$ and $m$ vertices with degree $\Delta=4$. So, by using Inequality (4), we have

$$
\operatorname{tvs}\left(C_{m} \triangleright_{o} C_{n}\right) \geq \max \left\{\left\lceil\frac{2+m(n-1)}{3}\right\rceil,\left\lceil\frac{2+m(n-1)+m}{5}\right\rceil\right\}=\left\lceil\frac{2+m(n-1)}{3}\right\rceil .
$$

So, we have

$$
\operatorname{tvs}\left(C_{m} \triangleright_{o} C_{n}\right) \geq\left\lceil\frac{m(n-1)+2}{3}\right\rceil .
$$




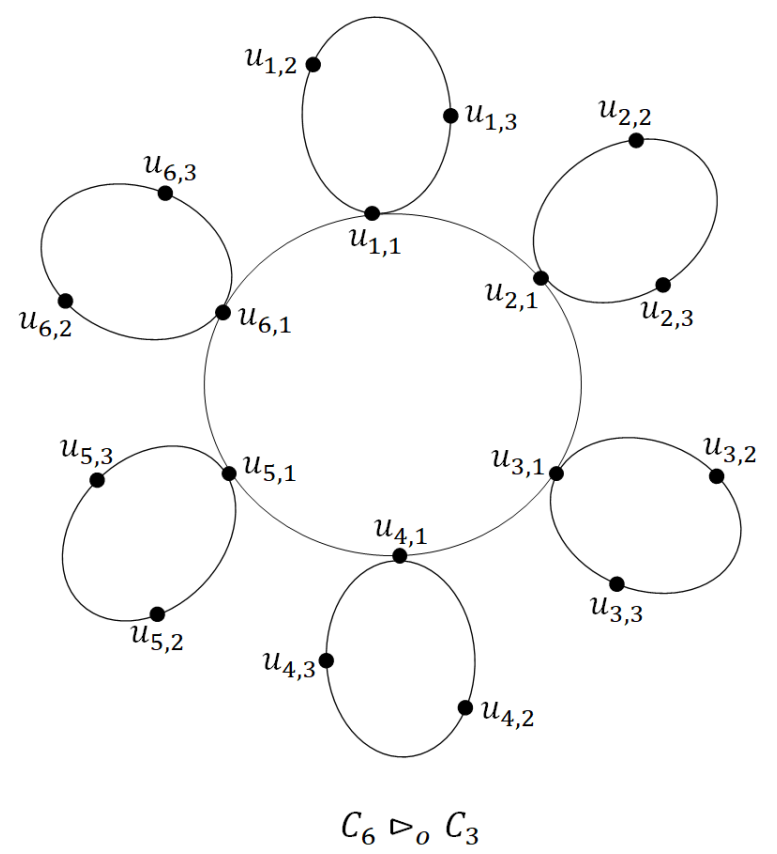

Figure 2. Comb product graph $C_{m} \triangleright_{o} C_{n}$ with $m=6$ and $n=3$

Next, we will show that

$$
\operatorname{tvs}\left(C_{m} \triangleright_{o} C_{n}\right) \leq\left\lceil\frac{m(n-1)+2}{3}\right\rceil .
$$

Define a total labeling $f: V\left(C_{m} \triangleright_{o} C_{n}\right) \cup E\left(C_{m} \triangleright_{o} C_{n}\right) \rightarrow\left\{1,2, \ldots,\left\lceil\frac{m(n-1)+2}{3}\right\rceil\right\}$ as follows.

$$
\begin{gathered}
f\left(u_{i, 1}\right)=\left\lceil\frac{m(n-1)+2}{3}\right\rceil, \text { for } 1 \leq i \leq m ; \\
f\left(u_{i, j}\right)=\left\lceil\frac{i(n-1)-(n-j)}{3}\right] \text { for } 1 \leq i \leq m, 2 \leq j \leq n ; \\
f\left(u_{i, j} u_{i, j+1}\right)=\left\lceil\frac{i(n-1)-(n-j-2)}{3}\right\rceil \text { for } 1 \leq i \leq m, 1 \leq j \leq n-1 ; \\
f\left(u_{i, n} u_{i, 1}\right)=\left\lceil\frac{i(n-1)+2}{3}\right\rceil \text { for } 1 \leq i \leq m ; \\
f\left(u_{i, 1} u_{i+1,1}\right)=\left\lceil\frac{m(n-1)+2}{3}\right\rceil \text { for } 1 \leq i \leq m-1 ; \\
f\left(u_{m, 1} u_{11}\right)=\left\lceil\frac{m(n-1)+2}{3}\right\rceil .
\end{gathered}
$$

From the labeling above, we have the weight of each vertex of $C_{m} \triangleright_{o} C_{n}$ as follows. 
1. For $1 \leq i \leq m$ and $j=1$, we devided the formula into three cases.

(a) Case $1:$ For $i=1$ and $j=1$,

$$
\begin{aligned}
w_{f}\left(u_{i, j}\right)= & w_{f}\left(u_{1,1}\right) \\
= & f\left(u_{1,1}\right)+f\left(u_{1,1} u_{2,1}\right)+f\left(u_{1,1} u_{m, 1}\right)+f\left(u_{1,1} u_{1,2}\right)+f\left(u_{1, n} u_{1,1}\right) \\
= & \left\lceil\frac{m(n-1)+2}{3}\right\rceil+\left\lceil\frac{m(n-1)+2}{3}\right\rceil+\left\lceil\frac{m(n-1)+2)}{3}\right\rceil+\left\lceil\frac{n-1-(n-1-2)}{3}\right\rceil \\
& +\left\lceil\frac{n-1+2}{3}\right\rceil \\
= & 3\left\lceil\frac{m(n-1)+2}{3}\right\rceil+\left\lceil\frac{2}{3}\right\rceil+\left\lceil\frac{n+1}{3}\right\rceil .
\end{aligned}
$$

(b) Case 2 : For $2 \leq i \leq m-1$ and $j=1$,

$$
\begin{aligned}
w_{f}\left(u_{i, j}\right)= & w_{f}\left(u_{i, 1}\right) \\
= & f\left(u_{i, 1}\right)+f\left(u_{i, 1} u_{i+1,1}\right)+f\left(u_{i-1, i} u_{i, 1}\right)+f\left(u_{i, 1} u_{i, 2}\right)+f\left(u_{i, n} u_{i, 1}\right) \\
= & \left\lceil\frac{m(n-1)+2}{3}\right\rceil+\left\lceil\frac{m(n-1)+2}{3}\right\rceil+\left\lceil\frac{m(n-1)+2)}{3}\right\rceil+\left\lceil\frac{i(n-1)-(n-1-2)}{3}\right\rceil \\
& +\left\lceil\frac{i(n-1)+2}{3}\right\rceil \\
= & 3\left\lceil\frac{m(n-1)+2}{3}\right\rceil+\left\lceil\frac{i(n-1)-(n-3)}{3}\right\rceil+\left\lceil\frac{i(n-1)+2}{3}\right\rceil .
\end{aligned}
$$

(c) Case 3 : For $i=m$ and $j=1$,

$$
\begin{aligned}
w_{f}\left(u_{i, j}\right)= & w_{f}\left(u_{m, 1}\right) \\
= & f\left(u_{m, 1}\right)+f\left(u_{m, 1} u_{1,1}\right)+f\left(u_{m-1, i} u_{m, 1}\right)+f\left(u_{m, 1} u_{m, 2}\right)+f\left(u_{m, n} u_{m, 1}\right) \\
= & \left\lceil\frac{m(n-1)+2}{3}\right\rceil+\left\lceil\frac{m(n-1)+2}{3}\right\rceil+\left\lceil\frac{m(n-1)+2)}{3}\right\rceil+\left\lceil\frac{m(n-1)-(n-1-2)}{3}\right\rceil \\
& +\left\lceil\frac{m(n-1)+2}{3}\right\rceil \\
= & 3\left\lceil\frac{m(n-1)+2}{3}\right\rceil+\left\lceil\frac{m(n-1)-(n-3)}{3}\right\rceil+\left\lceil\frac{m(n-1)+2}{3}\right\rceil .
\end{aligned}
$$

So, we have the general formula of $w_{f}\left(u_{i, j}\right)$, for $1 \leq i \leq m$ and $j=1$, as follows,

$$
w_{f}\left(u_{i, j}\right)=w_{f}\left(u_{i, 1}\right)=3\left\lceil\frac{m(n-1)+2}{3}\right\rceil+\left\lceil\frac{i(n-1)-(n-3)}{3}\right\rceil+\left\lceil\frac{i(n-1)+2}{3}\right\rceil .
$$

for $1 \leq i \leq m$. 
2. For $1 \leq i \leq m$ and $2 \leq j \leq n$, we devided the formula into two cases.

(a) Case 1: For $1 \leq i \leq m$ and $2 \leq j \leq n-1$,

$$
\begin{aligned}
& w_{f}\left(u_{i, j}\right)=f\left(u_{i, j}\right)+f\left(u_{i, j} u_{i, j+1}\right)+f\left(u_{i, j-1} u_{i, j}\right) \\
& =\left\lceil\frac{i(n-1)-(n-j)}{3}\right\rceil+\left\lceil\frac{i(n-1)-(n-j-2)}{3}\right\rceil+\left\lceil\frac{i(n-1)-(n-(j-1)-2)}{3}\right\rceil \\
& =\left\lceil\frac{i(n-1)-(n-j)}{3}\right\rceil+\left\lceil\frac{i(n-1)-(n-j-2)}{3}\right\rceil+\left\lceil\frac{i(n-1)-(n-j-1)}{3}\right\rceil \\
& =\left\lceil\frac{i(n-1)-(n-j)}{3}\right\rceil+\left\lceil\frac{i(n-1)-(n-j)+2}{3}\right\rceil+\left\lceil\frac{i(n-1)-(n-j)+1}{3}\right\rceil \\
& \left\{\begin{array}{l}
\left(\frac{i(n-1)-(n-j)}{3}\right)+\left(\frac{i(n-1)-(n-j)+3}{3}\right)+\left(\frac{i(n-1)-(n-j)+3}{3}\right) \\
\text { for } i(n-1)-(n-j) \equiv 0 \bmod 3
\end{array}\right. \\
& =\left\{\begin{array}{l}
\left(\frac{i(n-1)-(n-j)+2}{3}\right)+\left(\frac{i(n-1)-(n-j)+2}{3}\right)+\left(\frac{i(n-1)-(n-j)+2}{3}\right) \\
\text { for } i(n-1)-(n-j) \equiv 1 \bmod 3 ; \\
\left(\frac{i(n-1)-(n-j)+1}{3}\right)+\left(\frac{i(n-1)-(n-j)+4}{3}\right)+\left(\frac{i(n-1)-(n-j)+1}{3}\right) \\
\text { for } i(n-1)-(n-j) \equiv 2 \bmod 3 ;
\end{array}\right. \\
& =\left(\frac{3 i(n-1)-(n-j)+6}{3}\right) \\
& =i(n-1)-(n-j)+2 \text {. }
\end{aligned}
$$


(b) Case 2 : For $1 \leq i \leq m$ and $j=n$,

$$
\begin{aligned}
& w_{f}\left(u_{i, n}\right)= f\left(u_{i, n}\right)+f\left(u_{i, n} u_{1}\right)+f\left(u_{i, n-1} u_{i, n}\right) \\
&=\left.\frac{i(n-1)-(n-n)}{3}\right\rceil+\left\lceil\frac{i(n-1)+2}{3}\right\rceil+\left\lceil\frac{i(n-1)-(n-(n-1)-2)}{3}\right\rceil \\
&=\left.\frac{i(n-1)}{3}\right\rceil+\left\lceil\frac{i(n-1)+2}{3}\right\rceil+\left\lceil\frac{i(n-1)+1)}{3}\right\rceil \\
&=\left\{\begin{array}{l}
\left(\frac{i(n-1)}{3}\right)+\left(\frac{i(n-1)+3}{3}\right)+\left(\frac{i(n-1)+3}{3}\right) \\
\text { for } i(n-1) \equiv 0 \bmod 3 ; \\
\text { for } i(n-1) \equiv 1 \bmod 3 ; \\
\left(\frac{i(n-1)+1}{3}\right)+\left(\frac{i(n-1)+4}{3}\right)+\left(\frac{i(n-1)+1}{3}\right)
\end{array}\right) \\
& \text { for } i(n-1) \equiv 2 \bmod 3 ;
\end{aligned}
$$

So, we have the general formula of $w_{f}\left(u_{i, j}\right)$, for $1 \leq i \leq m$ and $2 \leq j \leq n$, as follows,

$$
w_{f}\left(u_{i, j}\right)=i(n-1)-(n-j)+2 \text {. }
$$

It will be shown that there are no two vertices with the same weight.

(a) It will be shown that $w_{f}\left(u_{i, 1}\right) \neq w_{f}\left(u_{k, 1}\right)$ for $i \neq k$ and $1 \leq i, k \leq m$.

Let $i=k+1$. It will be shown that $w_{f}\left(u_{i, 1}\right)>w_{f}\left(u_{k, 1}\right)$.

i. For $n=3$,

$$
\begin{aligned}
w_{f}\left(u_{i, 1}\right) & =3\left\lceil\frac{m(3-1)+2}{3}\right\rceil+\left\lceil\frac{i(3-1)-(3-3)}{3}\right\rceil+\left\lceil\frac{i(3-1)+2}{3}\right\rceil \\
& =3\left\lceil\frac{m(3-1)+2}{3}\right\rceil+\left\lceil\frac{(k+1)(3-1)-(3-3)}{3}\right\rceil+\left\lceil\frac{(k+1)(3-1)+2}{3}\right\rceil \\
& =3\left\lceil\frac{2 m+2}{3}\right\rceil+\left\lceil\frac{2 k+2}{3}\right\rceil+\left\lceil\frac{2 k+4}{3}\right\rceil \\
& >3\left\lceil\frac{2 m+2}{3}\right\rceil+\left\lceil\frac{2 k}{3}\right\rceil+\left\lceil\frac{2 k+2}{3}\right\rceil \\
& =w_{f}\left(u_{k, 1}\right) .
\end{aligned}
$$


ii. For $n \geq 4$,

$$
\begin{aligned}
w_{f}\left(u_{i, 1}\right) & =3\left\lceil\frac{m(n-1)+2}{3}\right]+\left\lceil\frac{i(n-1)-(n-3)}{3}\right\rceil+\left\lceil\frac{i(n-1)+2}{3}\right\rceil \\
& =3\left\lceil\frac{m(n-1)+2}{3}\right]+\left\lceil\frac{(k+1)(n-1)-(n-3)}{3}\right\rceil+\left\lceil\frac{(k+1)(n-1)+2}{3}\right\rceil \\
& =3\left\lceil\frac{m(n-1)+2}{3}\right]+\left\lceil\frac{k(n-1)+(n-1)-(n-3)}{3}\right\rceil+\left\lceil\frac{(k(n-1)+(n-1)+2)}{3}\right\rceil \\
& \geq 3\left[\frac{m(n-1)+2}{3}\right]+\left\lceil\frac{k(n-1)+(4-1)-(n-3)}{3}\right\rceil+\left\lceil\frac{k(n-1)+(4-1)+2}{3}\right\rceil \\
& =3\left\lceil\frac{m(n-1)+2}{3}\right]+\left\lceil\frac{k(n-1)+(3)-(n-3)}{3}\right\rceil+\left\lceil\frac{k(n-1)+(3)+2}{3}\right\rceil \\
& =3\left\lceil\frac{m(n-1)+2}{3}\right]+\left\lceil\frac{k(n-1)-(n-3)}{3}\right]+\left(\frac{3}{3}\right)+\left\lceil\frac{k(n-1)+2}{3}\right\rceil+\left(\frac{3}{3}\right) \\
& =3\left\lceil\frac{m(n-1)+2}{3}\right\rceil+\left\lceil\frac{k(n-1)-(n-3)}{3}\right\rceil+\left\lceil\frac{k(n-1)+2}{3}\right\rceil+2 \\
& =w_{f}\left(u_{k, 1}\right)+2 \\
& >w_{f}\left(u_{k, 1}\right) .
\end{aligned}
$$

So, it has been proven that $w_{f}\left(u_{i, 1}\right) \neq w_{f}\left(u_{k, 1}\right)$ for every $i \neq k$ and $1 \leq i, k \leq m$.

(b) It will be shown that $w_{f}\left(u_{i, j}\right) \neq w_{f}\left(u_{k, l}\right)$ for $i \neq k$ or $j \neq l, 1 \leq i, k \leq m$ and $2 \leq j, l \leq n$.

i. For $i>k$ and $j=l$,

$$
\begin{aligned}
w_{f}\left(u_{i, j}\right) & =i(n-1)-(n-j)+2 \\
& >k(n-1)-(n-j)+2 \\
& =k(n-1)-(n-l)+2 \\
& =w_{f}\left(u_{k, l}\right) .
\end{aligned}
$$

So, we have $w_{f}\left(u_{i, j}\right)>w_{f}\left(u_{k, l}\right)$ for $i>k$ and $j=l$.

ii. For $i>k$ and $j>l$,

$$
\begin{aligned}
w_{f}\left(u_{i, j}\right) & =i(n-1)-(n-j)+2 \\
& >k(n-1)-(n-l)+2 \\
& =w_{f}\left(u_{k, l}\right) .
\end{aligned}
$$

So, we have $w_{f}\left(u_{i, j}\right)>w_{f}\left(u_{k, l}\right)$ for $i>k$ and $j>l$.

iii. For $i>k$ and $j<l$,

$$
\begin{aligned}
w_{f}\left(u_{i, j}\right) & =i(n-1)-(n-j)+2 \\
& \geq(k+1)(n-1)-(n-j)+2 \\
& =k(n-1)+(n-1)-n+j+2 .
\end{aligned}
$$

Since $j \geq 2$,

$$
\begin{aligned}
k(n-1)+(n-1)-n+j+2 & \geq k(n-1)+(n-1)-n+2+2 \\
& =k(n-1)+n-n+3 .
\end{aligned}
$$


Since $l \leq n$,

$$
\begin{aligned}
k(n-1)+n-n+3 & \geq k(n-1)+l-n+3 . \\
& =k(n-1)-(n-l)+3 \\
& >k(n-1)-(n-l)+2 \\
& =w_{f}\left(u_{k, l}\right) .
\end{aligned}
$$

So, we have $w_{f}\left(u_{i, j}\right)>w_{f}\left(u_{k, l}\right)$ for $i>k$ and $j<l$.

So, it has been proven that $w_{f}\left(u_{i, j}\right) \neq w_{f}\left(u_{k, l}\right)$ for every $i \neq k$ or $j \neq l$ for $1 \leq i, k \leq$ $m$ and $2 \leq j, l \leq n$.

(c) It will be shown that $w_{f}\left(u_{i, 1}\right) \neq w_{f}\left(u_{k, l}\right)$ for $1 \leq i, k \leq m$ and $2 \leq l \leq n$.

Since $i \geq 1$, we have

$$
\begin{aligned}
w_{f}\left(u_{i, 1}\right) & =3\left\lceil\frac{m(n-1)+2}{3}\right\rceil+\left\lceil\frac{i(n-1)-(n-3)}{3}\right\rceil+\left\lceil\frac{i(n-1)+2}{3}\right\rceil . \\
& \geq 3\left\lceil\frac{m(n-1)+2}{3}\right\rceil+\left\lceil\frac{1(n-1)-(n-3)}{3}\right\rceil+\left\lceil\frac{1(n-1)+2}{3}\right\rceil . \\
& \geq 3\left\lceil\frac{m(n-1)+2}{3}\right\rceil+\left\lceil\frac{2}{3}\right\rceil+\left\lceil\frac{n+1}{3}\right\rceil .
\end{aligned}
$$

Since $3\left\lceil\frac{m(n-1)+2}{3}\right\rceil \geq 3\left(\frac{m(n-1)+2}{3}\right)$ and $n \geq 3$, we have

$$
\begin{aligned}
w_{f}\left(u_{i, 1}\right) & \geq 3\left\lceil\frac{m(n-1)+2}{3}\right\rceil+\left\lceil\frac{2}{3}\right\rceil+\left\lceil\frac{n+1}{3}\right\rceil . \\
& \geq 3\left(\frac{m(n-1)+2}{3}\right)+\left\lceil\frac{2}{3}\right\rceil+\left\lceil\frac{3+1}{3}\right\rceil . \\
& \geq(m(n-1)+2)+1+2 . \\
& \geq m(n-1)+5 .
\end{aligned}
$$

So, we have an inequality

$$
w_{f}\left(u_{i, 1}\right) \geq m(n-1)+5 \text {, }
$$

On the other hand, Since $1 \leq k \leq m$ and $2 \leq l \leq n$,

$$
\begin{aligned}
w_{f}\left(u_{k, l}\right) & =k(n-1)-n+l+2 \\
& \leq m(n-1)-n+n+2 \\
& \leq m(n-1)+2 . \\
& <m(n-1)+5 .
\end{aligned}
$$

So that, for $1 \leq k \leq m$ and $2 \leq l \leq n$, we have an inequality as follows,

$$
w_{f}\left(u_{k, l}\right)<m(n-1)+5
$$

From Inequatily (9) and (10), we have $w_{f}\left(u_{k, l}\right)<w_{f}\left(u_{i, 1}\right)$ for $1 \leq i, k \leq m$ and $2 \leq l \leq n$. 
From the three points above, we can conclude that from the labeling $f$, there are no two vertices with the same weight. So, $f$ is a vertex irregular total $\left\lceil\frac{m(n-1)+2}{3}\right\rceil$-labeling of $C_{m} \triangleright_{o} C_{n}$.

So we have Inequality (11),

$$
\operatorname{tvs}\left(C_{m} \triangleright_{o} C_{n}\right) \leq\left\lceil\frac{m(n-1)+2}{3}\right\rceil .
$$

By using Inequalities (6) and (11), we have an equation as follows,

$$
\operatorname{tvs}\left(C_{m} \triangleright_{o} C_{n}\right)=\left\lceil\frac{m(n-1)+2}{3}\right\rceil .
$$

The next theorem provides the total vertex irregularity strength of comb product between two stars.

Theorem 2.2. Let $S_{m}$ and $S_{n}$ be stars with order $m+1$ and $n+1$ respectively, and o be the center vertex of $S_{m}$. Then, for $m \geq 2$ and $n \geq 2$,

$$
\operatorname{tvs}\left(S_{m} \triangleright_{o} S_{n}\right)=\left\lceil\frac{n(m+1)+1}{2}\right\rceil .
$$

Proof. Let

$$
V\left(S_{m} \triangleright_{o} S_{n}\right)=\left\{u_{i, j} \mid 1 \leq i \leq m+1,1 \leq j \leq n+1\right\}
$$

and

$$
E\left(S_{m} \triangleright_{o} S_{n}\right)=\left\{u_{i, 1} u_{m+1,1} \mid 1 \leq i \leq m\right\} \cup\left\{u_{i, 1} u_{i, j} \mid 1 \leq i \leq m+1,2 \leq j \leq n+1 .\right\}
$$

An illustration of $S_{m} \triangleright_{o} S_{n}$ with $o$ be the center vertex in $S_{n}$ can be seen in Figure 3.

The $S_{m} \triangleright_{o} S_{n}$ graphs have $n(m+1)$ vertices with degree $\delta=1, m$ vertices with degree $n+1$, and one vertex with degree $n+m$. So, by using Inequality (4), we have

$$
\operatorname{tvs}\left(S_{m} \triangleright_{o} S_{n}\right) \geq \max \left\{\left\lceil\frac{n(m+1)+1}{2}\right\rceil,\left\lceil\frac{m n+n+2}{n+1}\right\rceil,\left\lceil\frac{m n+n+3}{n+m+1}\right\rceil\right\}=\left\lceil\frac{n(m+1)+1}{2}\right\rceil .
$$

So, we have

$$
\operatorname{tvs}\left(S_{m} \triangleright_{o} S_{n}\right) \geq\left\lceil\frac{n(m+1)+1}{2}\right\rceil .
$$

Next, we will show that

$$
\operatorname{tvs}\left(S_{m} \triangleright_{o} S_{n}\right) \leq\left\lceil\frac{n(m+1)+1}{2}\right\rceil .
$$

Define a total labeling $f: V\left(S_{m} \triangleright_{o} S_{n}\right) \cup E\left(S_{m} \triangleright_{o} S_{n}\right) \rightarrow\left\{1,2, \ldots,\left\lceil\frac{n(m+1)+1}{2}\right\rceil\right\}$ as follows. 


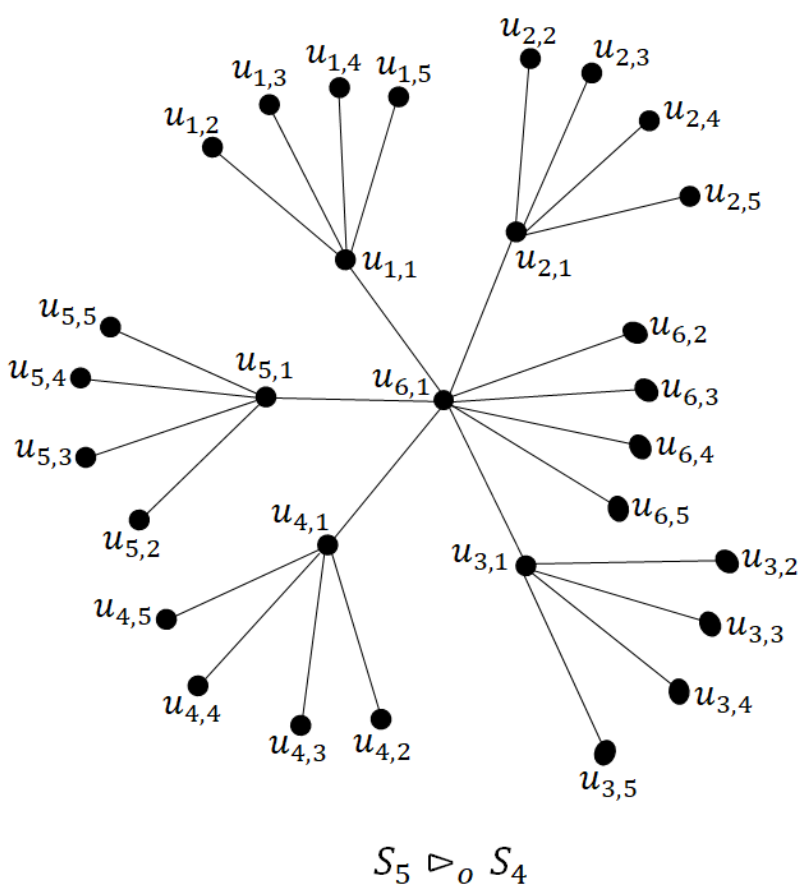

Figure 3. Comb product graph $S_{m} \triangleright_{o} S_{n}$ with $m=5$ and $n=4$

$$
\begin{gathered}
f\left(u_{i, 1}\right)=\left\lceil\frac{n(m+1)+1}{2}\right\rceil \text { for } 1 \leq i \leq m+1 \\
f\left(u_{i, j}\right)=\left\lceil\frac{n(i-1)+j-1}{2}\right\rceil, \text { for } 1 \leq i \leq m+1,2 \leq j \leq n+1 \\
f\left(u_{i, 1} u_{m+1,1}\right)=\left\lceil\frac{n(m+1)+1}{2}\right\rceil \text { for } 1 \leq i \leq m ; \\
f\left(u_{i, 1} u_{i, j}\right)=\left\lceil\frac{n(i-1)+j}{2}\right\rceil \text { for } 1 \leq i \leq m+1,2 \leq j \leq n+1 .
\end{gathered}
$$

From the labeling above, we have the weight of vertices of $S_{m} \triangleright_{o} S_{n}$ as follows. 
1. For $1 \leq i \leq m$ and $j=1$,

$$
\begin{aligned}
w_{f}\left(u_{i, j}\right)= & w_{f}\left(u_{i, 1}\right) \\
= & f\left(u_{i, 1}\right)+\sum_{j=2}^{n} f\left(u_{i, 1} u_{i, j}\right) \\
= & \left\lceil\frac{n(m+1)+1}{2}\right\rceil+\sum_{j=2}^{n}\left\lceil\frac{n(i-1)+j}{2}\right\rceil \\
= & \left\{\begin{array}{l}
2\left\lceil\frac{n(m+1)+1}{2}\right\rceil+n\left(\frac{n(i-1)}{2}\right)+\frac{n^{2}+4 n-1}{4} \text { for } n \text { is odd and } i \text { is odd; } \\
2\left\lceil\frac{n(m+1)+1}{2}\right\rceil+n\left(\frac{n(i-1)+1}{2}\right)+\frac{n^{2}+2 n+1}{4} \text { for } n \text { is odd and } i \text { is even; } \\
2\left\lceil\frac{n(m+1)+1}{2}\right\rceil+n\left(\frac{n(i-1)}{2}\right)+\frac{n^{2}+4 n}{4} \text { for } n \text { is even. }
\end{array}\right.
\end{aligned}
$$

2. For $i=m+1$ and $j=1$,

$$
\begin{aligned}
w_{f}\left(u_{i, j}\right)= & w_{f}\left(u_{m+1,1}\right) \\
= & f\left(u_{m+1,1}\right)+\sum_{j=2}^{n} f\left(u_{m+1,1} u_{m+1, j}\right)+\sum_{i=1}^{m} f\left(u_{i, 1} u_{m+1,1}\right) \\
= & \left\lceil\frac{n(m+1)+1}{2}\right\rceil+\sum_{j=2}^{n}\left\lceil\frac{n((m+1)-1)+j}{2}\right\rceil+\sum_{i=1}^{m}\left\lceil\frac{n(m+1)+1}{2}\right\rceil \\
= & \left\{\begin{array}{c}
(m+1)\left\lceil\frac{n(m+1)+1}{2}\right\rceil+n\left(\frac{n m+1}{2}\right)+\frac{n^{2}+2 n+1}{4} \text { for } n \text { is odd and } m \text { is odd; } \\
(m+1)\left\lceil\frac{n(m+1)+1}{2}\right\rceil+n\left(\frac{n m}{2}\right)+\frac{n^{2}+4 n-1}{4} \text { for } n \text { is odd and } m \text { is even; } \\
(m+1)\left\lceil\frac{n(m+1)+1}{2}\right\rceil+n\left(\frac{n m}{2}\right)+\frac{n^{2}+4 n}{4} \text { for } n \text { is even. }
\end{array}\right.
\end{aligned}
$$

3. For $1 \leq i \leq m+1$ and $2 \leq j \leq n+1$,

$$
\begin{aligned}
w_{f}\left(u_{i, j}\right)= & f\left(u_{i, j}\right)+f\left(u_{i, 1} u_{i, j}\right) \\
= & \left\lceil\frac{n(i-1)+j-1}{2}\right]+\left\lceil\frac{n(i-1)+j}{2}\right\rceil \\
= & \left\{\begin{array}{l}
\left(\frac{n(i-1)+j-1}{2}\right)+\left(\frac{n(i-1)+j+1}{2}\right) \text { for } n(i-1)+j \text { is odd; } \\
\left(\frac{n(i-1)+j}{2}\right)+\left(\frac{n(i-1)+j}{2}\right) \text { for } n(i-1)+j \text { is even; }
\end{array}\right. \\
= & n(i-1)+j .
\end{aligned}
$$

It will be shown that there are no two vertices with the same weight. 
1. It will be shown that $w_{f}\left(u_{i, 1}\right) \neq w_{f}\left(u_{k, 1}\right)$ for $i \neq k$ and $1 \leq i, k \leq m$.

Let $i=k+1$. It will be shown that $w_{f}\left(u_{i, 1}\right)>w_{f}\left(u_{k, 1}\right)$.

(a) For $i$ is odd, (then, $k$ is even), we have

$$
\begin{aligned}
w_{f}\left(u_{i, 1}\right) & =2\left[\frac{n(m+1)+1}{2}\right]+n\left(\frac{n(i-1)}{2}\right)+\frac{n^{2}+4 n-1}{4} \\
& =2\left[\frac{n(m+1)+1}{2}\right]+n\left(\frac{n(k)}{2}\right)+\frac{n^{2}+4 n-1}{4} . \\
& =2\left[\frac{n(m+1)+1}{2}\right]+n\left(\frac{n k-(n-1)}{2}\right)+\frac{n(n-1)}{2}+\frac{n^{2}+2 n+1}{4}+\frac{2 n-2}{4} . \\
& =2\left[\frac{n(m+1)+1}{2}\right]+n\left(\frac{n(k-1)+1}{2}\right)+\frac{n^{2}+2 n+1}{4}+\frac{n(n-1)}{2}+\frac{2 n-2}{4} . \\
& =w_{f}\left(u_{k, 1}\right)+\frac{n(n-1)}{2}+\frac{2 n-2}{4} .
\end{aligned}
$$

Since $n>2$, then $\frac{n(n-1)}{2}+\frac{2 n-2}{4}>1$. So,

$$
w_{f}\left(u_{i, 1}\right)>w_{f}\left(u_{k, 1}\right) .
$$

(b) For $i$ is even, (then, $k$ is odd), we have

$$
\begin{aligned}
w_{f}\left(u_{i, 1}\right) & =2\left[\frac{n(m+1)+1}{2}\right]+n\left(\frac{n(i-1)+1}{2}\right)+\frac{n^{2}+2 n+1}{4} \\
& =2\left[\frac{n(m+1)+1}{2}\right]+n\left(\frac{n(k)+1}{2}\right)+\frac{n^{2}+2 n+1}{4} . \\
& =2\left[\frac{n(m+1)+1}{2}\right]+n\left(\frac{n k+1}{2}\right)-\frac{2 n^{2}}{4}+\frac{n^{2}+4 n-1}{4}+\frac{2 n^{2}+2}{4}-\frac{2 n}{4} . \\
& =2\left[\frac{n(m+1)+1}{2}\right]+n\left(\frac{n k}{2}\right)+\frac{n}{2}-\frac{n^{2}}{2}+\frac{n^{2}+4 n-1}{4}+\frac{n^{2}+1}{2}-\frac{n}{2} . \\
& =2\left[\frac{n(m+1)+1}{2}\right]+n\left(\frac{n k}{2}\right)-\frac{n^{2}}{2}+\frac{n^{2}+4 n-1}{4}+\frac{n^{2}+1}{2} . \\
& =2\left\lceil\frac{n(m+1)+1}{2}\right]+n\left(\frac{n k-n}{2}\right)+\frac{n^{2}+4 n-1}{4}+\frac{n^{2}+1}{2} . \\
& =2\left\lceil\frac{n(m+1)+1}{2}\right]+n\left(\frac{n(k-1)}{2}\right)+\frac{n^{2}+4 n-1}{4}+\frac{n^{2}+1}{2} . \\
& =w_{f}\left(u_{k, 1}\right)+\frac{n^{2}+1}{2} .
\end{aligned}
$$

Since $n>2$, then $\frac{n^{2}+1}{2}>2$. So,

$$
w_{f}\left(u_{i, 1}\right)>w_{f}\left(u_{k, 1}\right) .
$$

So, it has been proven that $w_{f}\left(u_{i, 1}\right) \neq w_{f}\left(u_{k, 1}\right)$ for $i \neq k$ and $1 \leq i, k \leq m$.

2. It will be shown that $w_{f}\left(u_{i, 1}\right) \neq w_{f}\left(u_{m+1,1}\right)$ for $1 \leq i \leq m$.

For $1 \leq i \leq m$, we have Inequality (16),

$$
w_{f}\left(u_{i, 1}\right) \leq\left\{\begin{array}{l}
2\left\lceil\frac{n(m+1)+1}{2}\right\rceil+n\left(\frac{n(m-1)}{2}\right)+\frac{n^{2}+4 n-1}{4} \text { for } n \text { is odd and } m \text { is odd; } \\
2\left\lceil\frac{n(m+1)+1}{2}\right\rceil+n\left(\frac{n(m-1)+1}{2}\right)+\frac{n^{2}+2 n+1}{4} \text { for } n \text { is odd and } m \text { is even; } \\
2\left\lceil\frac{n(m+1)+1}{2}\right\rceil+n\left(\frac{n(m-1)}{2}\right)+\frac{n^{2}+4 n}{4} \text { for } n \text { is even. }
\end{array}\right.
$$


On the other hand, since $m>2$, we have Inequality (17),

$$
w_{f}\left(u_{m+1,1}\right)>\left\{\begin{array}{l}
2\left\lceil\frac{n(m+1)+1}{2}\right\rceil+n\left(\frac{n m+1}{2}\right)+\frac{n^{2}+2 n+1}{4} \text { for } n \text { is odd and } m \text { is odd; } \\
2\left\lceil\frac{n(m+1)+1}{2}\right\rceil+n\left(\frac{n m}{2}\right)+\frac{n^{2}+4 n-1}{4} \text { for } n \text { is odd and } m \text { is even; } \\
2\left\lceil\frac{n(m+1)+1}{2}\right\rceil+n\left(\frac{n m}{2}\right)+\frac{n^{2}+4 n}{4} \text { for } n \text { is even. }
\end{array}\right.
$$

From Inequality (16) and (17), we have

$$
w_{f}\left(u_{m+1,1}\right)>w_{f}\left(u_{i, 1}\right)
$$

for every $1 \leq i \leq m$.

3. It will be shown that $w_{f}\left(u_{i, j}\right) \neq w_{f}\left(u_{k, l}\right)$ for $i \neq k$ or $j \neq l, 1 \leq i, k \leq m+1$ and $2 \leq j, l \leq n+1$.

(a) For $i>k$ and $j=l$,

$$
\begin{aligned}
w_{f}\left(u_{i, j}\right) & =n(i-1)+j \\
& >n(k-1)+j \\
& =n(k-1)+l \\
& =w_{f}\left(u_{k, l}\right) .
\end{aligned}
$$

So, we have $w_{f}\left(u_{i, j}\right)>w_{f}\left(u_{k, l}\right)$ for $i>k$ and $j=l$.

(b) For $i>k$ and $j>l$,

$$
\begin{aligned}
w_{f}\left(u_{i, j}\right) & =n(i-1)+j \\
& >n(k-1)+j \\
& >n(k-1)+l \\
& =w_{f}\left(u_{k, l}\right) .
\end{aligned}
$$

So, we have $w_{f}\left(u_{i, j}\right)>w_{f}\left(u_{k, l}\right)$ for $i>k$ and $j>l$.

(c) For $i>k$ and $j<l$,

$$
\begin{aligned}
w_{f}\left(u_{i, j}\right) & =n(i-1)+j \\
\geq & n((k+1)-1)+j \\
& =n(k-1)+n+j .
\end{aligned}
$$

Since $j \geq 2$,

$$
n(k-1)+n+j \geq n(k-1)+n+2 .
$$

Since $n+1 \geq l$,

$$
\begin{aligned}
n(k-1)+n+2 & \geq n(k-1)+l+1 . \\
& >n(k-1)+l \\
& =w_{f}\left(u_{k, l}\right) .
\end{aligned}
$$

So, we have $w_{f}\left(u_{i, j}\right)>w_{f}\left(u_{k, l}\right)$ for $i>k$ and $j<l$. 
So, it has been proven that $w_{f}\left(u_{i, j}\right) \neq w_{f}\left(u_{k, l}\right)$ for every $i \neq k$ or $j \neq l$ for $1 \leq i, k \leq m+1$ and $2 \leq j, l \leq n+1$.

4. It will be shown that $w_{f}\left(u_{i, 1}\right) \neq w_{f}\left(u_{k, l}\right)$ for $1 \leq i, k \leq m+1$ and $2 \leq l \leq n+1$.

Since $k \leq m+1$ and $l \leq n+1$, we have

$$
\begin{aligned}
w_{f}\left(u_{k, l}\right) & =n(k-1)+l \\
& \leq n((m+1)-1)+(n+1) \\
& =n(m+1)+1 \\
& =2\left(\frac{n(m+1)+1}{2}\right) \\
& \leq 2\left\lceil\frac{n(m+1)+1}{2}\right\rceil .
\end{aligned}
$$

So, for $1 \leq k \leq m+1$ and $2 \leq l \leq n+1$, we have Inequality (19),

$$
w_{f}\left(u_{k, l}\right) \leq 2\left\lceil\frac{n(m+1)+1}{2}\right\rceil .
$$

On the other hand, for $1 \leq i \leq m$, we have

$$
w_{f}\left(u_{i, 1}\right)=\left\{\begin{array}{l}
2\left\lceil\frac{n(m+1)+1}{2}\right\rceil+n\left(\frac{n(i-1)}{2}\right)+\frac{n^{2}+4 n-1}{4} \text { for } n \text { is odd and } i \text { is odd; } \\
2\left\lceil\frac{n(m+1)+1}{2}\right\rceil+n\left(\frac{n(i-1)+1}{2}\right)+\frac{n^{2}+2 n+1}{4} \text { for } n \text { is odd and } i \text { is even; } \\
2\left\lceil\frac{n(m+1)+1}{2}\right\rceil+n\left(\frac{n(i-1)}{2}\right)+\frac{n^{2}+4 n}{4} \text { for } n \text { is even. }
\end{array}\right.
$$

Since $i \geq 1$, we have

$$
w_{f}\left(u_{i, 1}\right) \geq\left\{\begin{array}{l}
2\left\lceil\frac{n(m+1)+1}{2}\right\rceil+\frac{n^{2}+4 n-1}{4} \text { for } n \text { is odd and } i \text { is odd; } \\
2\left\lceil\frac{n(m+1)+1}{2}\right\rceil+n\left(\frac{1}{2}\right)+\frac{n^{2}+2 n+1}{4} \text { for } n \text { is odd and } i \text { is even; } \\
2\left\lceil\frac{n(m+1)+1}{2}\right\rceil+\frac{n^{2}+4 n}{4} \text { for } n \text { is even. }
\end{array}\right.
$$

Since $n>1$, then $\frac{n^{2}+4 n-1}{4}>1, n\left(\frac{1}{2}\right)+\frac{n^{2}+2 n+1}{4}>1$, and $\frac{n^{2}+4 n}{4}>1$. So, we have inequality as follows.

$$
w_{f}\left(u_{i, 1}\right)>2\left\lceil\frac{n(m+1)+1}{2}\right\rceil .
$$

From Inequality (19), (20), and (18), we have $w_{f}\left(u_{k, l}\right)<w_{f}\left(u_{i, 1}\right)<w_{f}\left(u_{m+1,1}\right)$ for $1 \leq i \leq m$, $1 \leq k \leq m+1$, and $2 \leq l \leq n+1$.

From the three points above, there are no two vertices with the same weight. So, $f$ is a vertex irregular total $\left\lceil\frac{n(m+1)+1}{2}\right\rceil$-labeling of $S_{m} \triangleright_{o} S_{n}$. So, we have inequality

$$
\operatorname{tvs}\left(S_{m} \triangleright_{o} S_{n}\right) \leq\left\lceil\frac{n(m+1)+1}{2}\right\rceil .
$$


From Inequality (13) and (21), we have Equation (22),

$$
\operatorname{tvs}\left(S_{m} \triangleright_{o} S_{n}\right)=\left\lceil\frac{n(m+1)+1}{2}\right\rceil .
$$

\section{Acknowledgement}

The research for this article was supported by Litapdimas Grant.

\section{References}

[1] M. Bača, S. Jendroľ, M. Miller, and J. Ryan, On irregular total labellings, Discrete Math. 307 (2007), 1378-1388.

[2] P. Majerski, and J. Przybylo, Total vertex irregularity strength of dense graphs, J. Graph Theory 76 (1) (2014), 34-41.

[3] Nurdin, E. T. Baskoro, A. N. M. Salman, and N. N. Goas, On the total vertex irregularity strength of trees, Discrete Math. 310 (2010), 3043-3048.

[4] Nurdin, E. T. Baskoro, A. N. M. Salman, and N.N. Goas, On the total vertex irregular labeling for several types of trees, Utilitas Math. 83 (2010), 277-290.

[5] Nurdin, A. N. M. Salman, N. N. Goas, and E. T. Baskoro, On the total vertex-irregular strength of a disjoint of t copies of a path, J. Combin. Math. 71 (2009), 227-233.

[6] J. Przybylo, Liniear bound on the irregularity strength and the total vertex irregularity strength of graphs, SIAM J. Discrete Math. 23 (2009), 511-516

[7] R. Ramdani, A. N. M. Salman, H. Assiyatun, and A. Semaničová-Feňovcičová, M. Bača, On the total irregularity strength of disjoint union of arbitrary graphs, Math. Rep. 18(68), 4 (2016), 469-482.

[8] R. Ramdani and M. A. Ramdhani, Total vertex irregularity strength of comb product of two cycles, MATEC Web Conf. 197, 01007 (2018).

[9] K. Wijaya and Slamin, Total vertex irregular labeling of wheels, fans, suns, and friendship graphs, J. Combin. Math. Combin. Comput. 65 (2008), 103-112.

[10] K. Wijaya, Slamin, Surahmat, and S. Jendroľ, Total vertex irregular labeling of complete bipartit graphs, J. Combin. Math. Combin. Comput. 55 (2005), 129-136. 